\title{
An Overdetermined Problem for an Elliptic Equation
}

\author{
by \\ Robert DALMASSO
}

\begin{abstract}
We consider the following overdetermined boundary value problem: $\Delta u+\lambda u+\mu=0$ in $\Omega, u=0$ on $\partial \Omega$ and $\partial u / \partial n=c$ on $\partial \Omega$, where $c \neq 0, \lambda$ and $\mu$ are real constants and $\Omega \subset \mathbb{R}^{2}$ is a smooth bounded convex open set. We first show that it may happen that the problem has no solution. Then we study the existence of solutions for a wide class of domains.
\end{abstract}

2010 Mathematics Subject Classification: 35J05, 35R30.

Keywords: overdetermined elliptic boundary value problem, Schiffer property, Schiffer conjecture.

\section{§1. Introduction}

Let $\Omega \subset \mathbb{R}^{2}$ be a smooth bounded simply-connected open set. We consider solutions of the following overdetermined elliptic boundary value problem:

$$
\begin{array}{rlrl}
\Delta u+\lambda u+\mu & =0 & & \text { in } \Omega, \\
u=0 & & \text { on } \partial \Omega, \\
\frac{\partial u}{\partial n}=c & & \text { on } \partial \Omega,
\end{array}
$$

where $\lambda, \mu$ and $c$ are real constants and $\partial / \partial n$ is the outward normal derivative.

If $c=0$ and $\mu \neq 0$ (or equivalently $\mu=1$ ) we get as a special case Schiffer's problem (Yau [18, p. 688, problem 80]). If $\mu=0$ and $c \neq 0$ the problem was posed by Berenstein [1].

In 1981 Williams 16 proved that if $\partial \Omega$ is Lipschitz and (1.1)-(1.3) has a solution for $c=0$ and $\mu=1$, then $\partial \Omega$ is real analytic. In 2002 Williams [17.

Communicated by S. Mukai. Received April 23, 2007. Revised February 8, 2010.

R. Dalmasso: Laboratoire Jean Kuntzmann, Equipe EDP, Domaine universitaire, 51 rue des Mathématiques, BP 53, 38041 Grenoble Cedex 9, France;

e-mail: robert.dalmasso@imag.fr

(C) 2010 Research Institute for Mathematical Sciences, Kyoto University. All rights reserved. 
proved that if $\partial \Omega$ is $C^{1}$ and (1.1)-(1.3) has a nonconstant solution $u \in C^{2}(\bar{\Omega})$, then $\partial \Omega$ is real analytic. In both cases the result holds in any dimension.

The following conjecture is stated in [17] (see also [10]):

Conjecture. Assume that $\partial \Omega$ is Lipschitz. If (1.1)-(1.3) has a nonconstant solution for some real constants $\lambda, \mu$ and $c$, then $\Omega$ is a disk.

Assume that $\Omega$ is the unit disk. Let $J_{z}$ denote the $z$-th Bessel function. For any $\lambda>0$ such that $\sqrt{\lambda}$ is not a zero of $J_{1}$ we define the function

$$
u_{\lambda}(x)=\frac{J_{0}(\sqrt{\lambda}|x|)-J_{0}(\sqrt{\lambda})}{\sqrt{\lambda} J_{1}(\sqrt{\lambda})}, \quad|x|<1 .
$$

For $\lambda<0$ we define the function

$$
u_{\lambda}(x)=-i \frac{J_{0}(i \sqrt{-\lambda}|x|)-J_{0}(i \sqrt{-\lambda})}{\sqrt{-\lambda} J_{1}(i \sqrt{-\lambda})}, \quad|x|<1 .
$$

We recall that $J_{1}$ has only real zeros [14, pp. 482-483]. We easily verify that $u_{\lambda}$ is a solution of (1.1)-(1.3) with $c=-1$ and $\mu=\mu_{\lambda}$ given by

$$
\mu_{\lambda}= \begin{cases}\frac{\sqrt{\lambda} J_{0}(\sqrt{\lambda})}{J_{1}(\sqrt{\lambda})}, & \lambda>0 \\ \frac{i \sqrt{-\lambda} J_{0}(i \sqrt{-\lambda})}{J_{1}(i \sqrt{-\lambda})}, & \lambda<0 .\end{cases}
$$

The functions $u_{\lambda}$ have a removable singularity at $\lambda=0$ and the corresponding solution is

$$
u_{0}(x)=\frac{1}{2}\left(1-|x|^{2}\right), \quad|x|<1 .
$$

Therefore, when $\Omega$ is the unit disk, there is a continuum of coefficient pairs $\left(\lambda, \mu_{\lambda}\right)$ and $u_{\lambda}$ which solve (1.1)-(1.3) with $c \neq 0$. Notice that when $\lambda>0$ is such that $J_{0}(\sqrt{\lambda})=0$ we have $\mu_{\lambda}=0$. Then the corresponding Dirichlet problem has infinitely many solutions giving rise to the same constant normal derivative on the boundary. Berenstein 1] proved the following converse.

Proposition 1.1. Let $\Omega$ be a simply-connected bounded open subset of $\mathbb{R}^{2}$ with $C^{2, \varepsilon}$ boundary $(\varepsilon>0)$. Assume that (1.1)-(1.3) with $\mu=0$ and $c \neq 0$ has a solution for infinitely many $\lambda$. Then $\Omega$ is a disk.

A smooth bounded simply-connected open set $\Omega \subset \mathbb{R}^{2}$ is said to have the Schiffer property if (for any $\lambda$ ) the only solution to (1.1)-(1.3) with $c=0$ is the trivial solution $u=0$ (corresponding to $\mu=0$ ). It is well known that disks do not have the Schiffer property. Indeed, let $\lambda>0$ be such that $J_{1}(\sqrt{\lambda})=0$. Then the 
function

$$
v_{\lambda}(x)=\frac{1}{\lambda}\left(\frac{J_{0}(\sqrt{\lambda}|x|)}{J_{0}(\sqrt{\lambda})}-1\right), \quad|x|<1,
$$

satisfies (1.1)-(1.3) with $\mu=1$ and $c=0$ when $\Omega$ is the unit disk. Berenstein [1] proved the following converse.

Proposition 1.2. Let $\Omega$ be a simply-connected bounded open subset of $\mathbb{R}^{2}$ with $C^{2, \varepsilon}$ boundary $(\varepsilon>0)$. Assume that (1.1)-(1.3) with $\mu=1$ and $c=0$ has a solution for infinitely many $\lambda$. Then $\Omega$ is a disk.

Remark 1. An elementary proof of Propositions 1.1 and 1.2 is given in [5] in the particular case where $\Omega$ is a convex set with positive curvature.

The Schiffer conjecture asserts that disks are the only smooth bounded simplyconnected open sets for which (1.1)-(1.3) with $\mu=1$ and $c=0$ has a solution for even a single value of $\lambda$. Williams [15] established that for smooth bounded simplyconnected open sets the Schiffer property is equivalent to the Pompeiu property. We shall not define the latter, instead we refer the reader to the bibliographic survey of the Pompeiu problem ([19]). Wide classes of smooth bounded simplyconnected open sets in $\mathbb{R}^{2}$ having the Schiffer property were studied in [9] and the references therein. In [3] and 4] we gave some elementary results allowing us to exhibit very simple examples of planar domains having the Schiffer property. However, when $c \neq 0$, we do not know of any example supporting the above conjecture, even in the particular case studied by Berenstein. We first examine this problem.

Proposition 1.3. There exist smooth bounded simply-connected open sets $\Omega \subset \mathbb{R}^{2}$ such that, for any fixed constant $c \neq 0,(1.1)-(1.3)$ has no solution.

The width of a convex planar domain in a given direction is the distance between two parallel supporting lines perpendicular to that direction. A set of constant width has the same width in all directions. Clearly disks have constant width. However there are plenty of smooth domains which have constant width but which are not disks: see [2], [5], [11] and [13].

Now we can state our main result.

Theorem 1.1. Let $\Omega \subset \mathbb{R}^{2}$ be a bounded convex open set satisfying the following conditions:

(i) $\partial \Omega$ is a $C^{\infty}$ curve with positive curvature;

(ii) $\Omega$ has the Schiffer property;

(iii) $\Omega$ is not of constant width. 
Let $c$ be a nonzero constant. Then there exist at most finitely many different pairs of coefficients $\left(\lambda_{m}, \mu_{m}\right) \in \mathbb{R}^{2}$ such that the Cauchy problem

$$
\Delta u+\lambda_{m} u+\mu_{m}=0 \quad \text { in } \Omega, \quad u=0 \quad \text { and } \quad \frac{\partial u}{\partial n}=c \quad \text { on } \partial \Omega,
$$

has a solution.

Remark 2. A true ellipse satisfies the conditions of Theorem 1.1. Indeed, it is well known that a true ellipse has the Schiffer property: a very simple proof is given in 6].

\section{§2. Preliminaries}

We assume first that $\Omega \subset \mathbb{R}^{2}$ is a bounded simply-connected open set with $C^{\infty}$ boundary $\partial \Omega$. Let $x=x(s)=\left(x_{1}(s), x_{2}(s)\right), s \in[0, L]$, be a parametrization of $\partial \Omega$ by arc length. We denote by $\tau(s)=\left(\tau_{1}(s), \tau_{2}(s)\right)$ the tangent to $\partial \Omega$ at $x(s)$ and by $\nu(s)=\left(\nu_{1}(s), \nu_{2}(s)\right)$ the exterior normal to $\partial \Omega$ at $x(s)$. We have

$$
\tau_{1}(s)=x_{1}^{\prime}(s), \quad \tau_{2}(s)=x_{2}^{\prime}(s), \quad s \in[0, L],
$$

and

$$
\nu_{1}(s)=x_{2}^{\prime}(s), \quad \nu_{2}(s)=-x_{1}^{\prime}(s), \quad s \in[0, L] .
$$

The Frenet formulas are

$$
x^{\prime \prime}(s)=-\kappa(s) \nu(s), \quad \nu^{\prime}(s)=\kappa(s) x^{\prime}(s), \quad s \in[0, L],
$$

where $\kappa=\kappa(s)$ is the curvature.

Now suppose that there exists $u \in C^{\infty}(\bar{\Omega})$ satisfying $(1.1)-(1.3)$. We shall use some formulas established in a more general situation [7, Lemma 2.5 p. 101 and Lemma 2.6 p. 104] (see also [5]).

\section{Lemma 2.1.}

(1) We have

$$
\left(\lambda c^{2}+\mu^{2}\right) \int_{0}^{L}\left(x_{1} \nu_{2}-x_{2} \nu_{1}\right)\left(\nu_{1}+i \nu_{2}\right)^{2} d s+A \mu+B=0,
$$

where $A$ and $B$ are independent of $\lambda$ and $\mu$. Moreover

$$
\begin{aligned}
& A=2 i c \int_{0}^{L}\left(2 \kappa\left(x \cdot \nu+i\left(x_{1} \nu_{2}-x_{2} \nu_{1}\right)\right)-1\right)\left(\nu_{1}+i \nu_{2}\right)^{2} d s \\
& B=-2 i c^{2} \int_{0}^{L} \kappa\left(\kappa\left(x \cdot \nu+i\left(x_{1} \nu_{2}-x_{2} \nu_{1}\right)\right)-1\right)\left(\nu_{1}+i \nu_{2}\right)^{2} d s .
\end{aligned}
$$


(2) Let $k=2 p$ with $p \geq 2$. We have

$$
\begin{aligned}
0= & (-1)^{p} \lambda^{p-1}\left(\lambda c^{2}+\mu^{2}\right) \int_{0}^{L}\left(x_{1} \nu_{2}-x_{2} \nu_{1}\right)\left(\nu_{1}+i \nu_{2}\right)^{k} d s \\
& +\mu^{2} \sum_{j=2}^{p} A_{j, k} \lambda^{p-j}+\mu \sum_{j=1}^{p} B_{j, k} \lambda^{p-j}+\sum_{j=1}^{p} C_{j, k} \lambda^{p-j},
\end{aligned}
$$

where $A_{j, k}, B_{j, k}$ and $C_{j, k}$ are independent of $\lambda$ and $\mu$. Moreover

$$
B_{1, k}=(-1)^{p} i k c \int_{0}^{L}\left(\kappa\left(2 x \cdot \nu+i k\left(x_{1} \nu_{2}-x_{2} \nu_{1}\right)\right)-1\right)\left(\nu_{1}+i \nu_{2}\right)^{k} d s .
$$

\section{Lemma 2.2.}

(1) We have

$$
\left(\lambda c^{2}+\mu^{2}\right) \int_{0}^{L}\left(\nu_{1}+i \nu_{2}\right)^{3} d s+a \mu+b=0,
$$

where $a$ and $b$ are independent of $\lambda$ and $\mu$. Moreover

$$
a=-8 c \int_{0}^{L} \kappa\left(\nu_{1}+i \nu_{2}\right)^{3} d s \quad \text { and } \quad b=4 c^{2} \int_{0}^{L} \kappa^{2}\left(\nu_{1}+i \nu_{2}\right)^{3} d s .
$$

(2) Let $k=2 p+1$ with $p \geq 2$. We have

$$
\begin{aligned}
0= & (-1)^{p} \lambda^{p-1}\left(\lambda c^{2}+\mu^{2}\right) \int_{0}^{L}\left(\nu_{1}+i \nu_{2}\right)^{k} d s \\
& +\mu^{2} \sum_{j=2}^{p} \alpha_{j, k} \lambda^{p-j}+\mu \sum_{j=1}^{p} \beta_{j, k} \lambda^{p-j}+\sum_{j=1}^{p} \gamma_{j, k} \lambda^{p-j},
\end{aligned}
$$

where $\alpha_{j, k}, \beta_{j, k}$ and $\gamma_{j, k}$ are independent of $\lambda$ and $\mu$. Moreover

$$
\begin{aligned}
\alpha_{2, k} & =(-1)^{p} \frac{2}{3}(p-1) p(p+1)(p+2) \int_{0}^{L} \kappa^{2}\left(\nu_{1}+i \nu_{2}\right)^{k} d s \\
\beta_{1, k} & =(-1)^{p-1}\left(k^{2}-1\right) c \int_{0}^{L} \kappa\left(\nu_{1}+i \nu_{2}\right)^{k} d s \\
\gamma_{1, k} & =(-1)^{p} \frac{2}{3} p(p+1)\left(p^{2}+p+1\right) c^{2} \int_{0}^{L} \kappa^{2}\left(\nu_{1}+i \nu_{2}\right)^{k} d s \\
\beta_{2,5} & =-16 c \int_{0}^{L} \kappa^{3}\left(\nu_{1}+i \nu_{2}\right)^{5} d s \\
\gamma_{2,5} & =-16 c^{2}\left(\frac{8}{3} \int_{0}^{L} \kappa^{4}\left(\nu_{1}+i \nu_{2}\right)^{5} d s+\int_{0}^{L} \kappa^{\prime 2}\left(\nu_{1}+i \nu_{2}\right)^{5} d s\right) .
\end{aligned}
$$


Remark 3. In [7], $B, \beta_{2,5}$ and $\gamma_{2,5}$ are not given explicitly, but they can be easily obtained from the proof.

Lemma 2.3. Assume that $\Omega$ has the Schiffer property. Given any $c \in \mathbb{R} \backslash\{0\}$ and $\lambda \in \mathbb{R}$, there exists at most one $\mu \in \mathbb{R}$ such that the Cauchy problem

$$
\Delta v+\lambda v+\mu=0 \quad \text { in } \Omega, \quad v=0 \quad \text { and } \quad \frac{\partial v}{\partial n}=c \quad \text { on } \partial \Omega,
$$

has a solution.

Proof. Suppose there exist $c \in \mathbb{R} \backslash\{0\}$ and $\lambda \in \mathbb{R}$ for which the above problem has a solution for two different values $\mu_{1}$ and $\mu_{2}$. We denote by $v_{1}$ and $v_{2}$ two solutions corresponding to $\mu_{1}$ and $\mu_{2}$ respectively. The function $u=\left(v_{1}-v_{2}\right) /\left(\mu_{1}-\mu_{2}\right)$ satisfies (1.1)-(1.3) with $\mu=1$ and $c=0$ and we reach a contradiction.

Assume moreover that $\partial \Omega$ has positive curvature and that $0 \in \Omega$. Since the curve $\partial \Omega$ turns continuously, to each point $x=x(s) \in \partial \Omega$ we can associate a unique $\theta$ (modulo $2 \pi$ ) and $\theta$ describes a complete circuit $0 \leq \theta \leq 2 \pi$ as $0 \leq s \leq L$. For each angle $\theta, 0 \leq \theta<2 \pi$, let $h(\theta)$ denote the distance from the origin to the supporting line of $\Omega$ with outward normal $\nu=(\cos \theta, \sin \theta)$. We have

$$
h(\theta)=x \cdot \nu,
$$

and $h$ has period $2 \pi$. From the Serret-Frenet formulas we can derive the following second order ordinary differential equation involving the support function $h$ and the radius of curvature $\rho$ :

$$
h(\theta)+h^{\prime \prime}(\theta)=\rho(\theta) .
$$

When $0 \notin \Omega$, the support function is defined in the following way. By translation there exists $a=\left(a_{1}, a_{2}\right) \in \mathbb{R}^{2}$ such that $0 \in \tilde{\Omega}=a+\Omega$. If $\tilde{h}$ denotes the support function of $\tilde{\Omega}$ we have

$$
h(\theta)=-a_{1} \cos \theta-a_{2} \sin \theta+\tilde{h}(\theta) .
$$

We refer the reader to Flanders [8] and the references therein for a detailed discussion.

For any $f:[0,2 \pi] \rightarrow \mathbb{C}$ such that $f \in L^{2}[0,2 \pi]$ we denote by

$$
c_{n}(f)=\frac{1}{2 \pi} \int_{0}^{2 \pi} f(\theta) e^{-i n \theta} d \theta, \quad n \in \mathbb{Z},
$$

the Fourier coefficients. 


\section{$\S 3$. Proof of Proposition 1.3}

Let $r>32$. Define

$$
h(\theta)=r+\cos 3 \theta+\cos 5 \theta, \quad 0 \leq \theta \leq 2 \pi .
$$

Then $h$ is of class $C^{\infty}$ and has period $2 \pi$. Since

$$
\rho(\theta)=r-8 \cos 3 \theta-24 \cos 5 \theta>0, \quad 0 \leq \theta \leq 2 \pi,
$$

$h$ must be the support function of a convex set $\Omega$.

Lemma 3.1. Let $\Omega$ be as above. Assume that there exist $c \neq 0, \lambda, \mu \in \mathbb{R}$ and $u \in C^{\infty}(\bar{\Omega})$ satisfying (1.1)-(1.3). With the notations of Lemmas 2.1 and $2.2, b / c^{2}$ and $B / c^{2}$ depend only on $r$ and we have

$\int_{0}^{L}\left(x_{1} \nu_{2}-x_{2} \nu_{1}\right)\left(\nu_{1}+i \nu_{2}\right)^{2} d s=16 i \pi, \quad \int_{0}^{L}\left(\nu_{1}+i \nu_{2}\right)^{3} d s=-8 \pi, \quad A=a=0$,

and

$$
\frac{B}{c^{2}}=-\frac{2^{6} \cdot 7 i \pi}{r^{2}}+o\left(\frac{1}{r^{2}}\right), \quad \frac{b}{c^{2}}=\frac{2^{5} \pi}{r^{2}}+o\left(\frac{1}{r^{3}}\right), \quad \text { as } r \rightarrow+\infty .
$$

Proof. We have

$$
\begin{gathered}
\int_{0}^{L}\left(\nu_{1}+i \nu_{2}\right)^{3} d s=\int_{0}^{2 \pi} \rho(\theta) e^{3 i \theta} d \theta=-8 \pi \\
\int_{0}^{L}\left(x_{1} \nu_{2}-x_{2} \nu_{1}\right)\left(\nu_{1}+i \nu_{2}\right)^{2} d s=-\int_{0}^{2 \pi} h^{\prime}(\theta) \rho(\theta) e^{2 i \theta} d \theta
\end{gathered}
$$

and

$$
\begin{aligned}
-\int_{0}^{2 \pi} h^{\prime}(\theta) \rho(\theta) e^{2 i \theta} d \theta= & \int_{0}^{2 \pi}(3 \sin 3 \theta+5 \sin 5 \theta)(r-8(\cos 3 \theta+3 \cos 5 \theta)) e^{2 i \theta} d \theta \\
= & -2^{3} \cdot 3^{2} \int_{0}^{2 \pi} e^{2 i \theta} \sin 3 \theta \cos 5 \theta d \theta \\
& -2^{3} \cdot 5 \int_{0}^{2 \pi} e^{2 i \theta} \sin 5 \theta \cos 3 \theta d \theta=16 i \pi, \\
a= & -8 c \int_{0}^{2 \pi} e^{3 i \theta} d \theta=0, \\
A= & 2 i c \int_{0}^{2 \pi}\left(2\left(h(\theta)-i h^{\prime}(\theta)\right)-\rho(\theta)\right) e^{2 i \theta} d \theta=0 .
\end{aligned}
$$

Next as $r \rightarrow+\infty$ we have

$$
\frac{B}{c^{2}}=-2 i \int_{0}^{2 \pi} \frac{h(\theta)-i h^{\prime}(\theta)}{\rho(\theta)} e^{2 i \theta} d \theta
$$




$$
\begin{aligned}
= & -\frac{2 i}{r} \sum_{n \geq 0} \frac{8^{n}}{r^{n}} \int_{0}^{2 \pi}(r+\cos 3 \theta+\cos 5 \theta+3 i \sin 3 \theta+5 i \sin 5 \theta) \\
& \times(\cos 3 \theta+3 \cos 5 \theta)^{n} e^{2 i \theta} d \theta \\
= & -\frac{2^{4} i}{r^{2}} \int_{0}^{2 \pi}\left(2^{2} \cdot 13 \cos 3 \theta \cos 5 \theta+3^{2} i \sin 3 \theta \cos 5 \theta\right. \\
& +5 i \cos 3 \theta \sin 5 \theta) e^{2 i \theta} d \theta+o\left(\frac{1}{r^{2}}\right) \\
= & -\frac{2^{6} \cdot 7 i \pi}{r^{2}}+o\left(\frac{1}{r^{2}}\right),
\end{aligned}
$$

and

$$
\begin{aligned}
\frac{b}{c^{2}} & =4 \int_{0}^{2 \pi} \frac{e^{3 i \theta}}{\rho(\theta)} d \theta=\frac{4}{r} \sum_{n \geq 0} \frac{8^{n}}{r^{n}} \int_{0}^{2 \pi}(\cos 3 \theta+3 \cos 5 \theta)^{n} e^{3 i \theta} d \theta \\
& =\frac{2^{5}}{r^{2}} \int_{0}^{2 \pi} e^{3 i \theta} \cos 3 \theta d \theta+o\left(\frac{1}{r^{3}}\right)=\frac{2^{5} \pi}{r^{2}}+o\left(\frac{1}{r^{3}}\right) .
\end{aligned}
$$

Now we can complete the proof of Proposition 1.3. Choose $\Omega$ as in Lemma 3.1 with $r$ sufficiently large. Using Lemmas 2.1(1), 2.2(1) and 3.1 we get

$$
\lambda+\frac{\mu^{2}}{c^{2}}-\frac{28}{r^{2}}+o\left(\frac{1}{r^{2}}\right)=0 \text { and } \lambda+\frac{\mu^{2}}{c^{2}}-\frac{4}{r^{2}}+o\left(\frac{1}{r^{3}}\right)=0,
$$

and we obtain a contradiction.

Remark 4. For any $r>32, \Omega$ has the Schiffer property and $\Omega$ is of constant width. Indeed, if (1.1)-(1.3) has a nontrivial solution when $c=0$, then Lemma 2.2(1) implies that

$$
\int_{0}^{L}\left(\nu_{1}+i \nu_{2}\right)^{3} d s=0
$$

and we have a contradiction. On the other hand $h(\theta)+h(\theta+\pi)=2 r$ for $\theta \in[0,2 \pi]$, hence $\Omega$ is of constant width.

Remark 5 . Notice that in the case considered by Berenstein $(\mu=0)$ we can easily give examples of sets $\Omega$ satisfying the conclusion of Proposition 1.3 and such that:

(i) $\Omega$ have the Schiffer property;

(ii) $\Omega$ are not of constant width.

Indeed, let $r>11$ and define

$$
h(\theta)=r+\cos 2 \theta+\cos 3 \theta, \quad \theta \in[0,2 \pi] .
$$


Then $h$ is of class $C^{\infty}$ and has period $2 \pi$. Since

$$
\rho(\theta)=r-3 \cos 2 \theta-8 \cos 3 \theta>0, \quad 0 \leq \theta \leq 2 \pi,
$$

$h$ must be the support function of a convex set $\Omega$. As in Lemma 3.1 we have

$$
\int_{0}^{L}\left(x_{1} \nu_{2}-x_{2} \nu_{1}\right)\left(\nu_{1}+i \nu_{2}\right)^{2} d s=2 i r \pi, \quad \int_{0}^{L}\left(\nu_{1}+i \nu_{2}\right)^{3} d s=-8 \pi,
$$

and

$$
\frac{B}{c^{2}}=-\frac{4 i \pi}{r}+o\left(\frac{1}{r^{2}}\right), \quad \frac{b}{c^{2}}=\frac{2^{5} \pi}{r^{2}}+o\left(\frac{1}{r^{2}}\right), \quad \text { as } r \rightarrow+\infty .
$$

Then using Lemmas 2.1(1) and 2.2(1) we obtain

$$
\lambda-\frac{2}{r^{2}}+o\left(\frac{1}{r^{3}}\right)=0 \quad \text { and } \quad \lambda-\frac{4}{r^{2}}+o\left(\frac{1}{r^{3}}\right)=0,
$$

and we have a contradiction. On the other hand, since

$$
\int_{0}^{L}\left(\nu_{1}+\nu_{2}\right)^{3} d s \neq 0 \quad \text { and } \quad c_{2}(h) \neq 0,
$$

(i) and (ii) are satisfied.

In fact we could also treat the case $\mu \neq 0$. From Lemmas 2.1(1) and 2.2(1) we get two equations for $\lambda, \mu / c$ and $\mu^{2} / c^{2}$. Then Lemma 2.2(2) with $p=2$ gives a third equation:

$$
\alpha_{2,5} \frac{\mu^{2}}{c^{2}}+\frac{\beta_{2,5}}{c} \frac{\mu}{c}+\frac{\gamma_{1,5}}{c^{2}} \lambda+\frac{\gamma_{2,5}}{c^{2}}=0 .
$$

However, to get a contradiction requires tedious calculations; we leave the details to the reader.

\section{$\S 4$. Proof of Theorem 1.1}

Let $c \neq 0$ be a given constant. Assume that (1.1)-(1.3) has a solution corresponding to a sequence of infinitely many different pairs $\left(\lambda_{m}, \mu_{m}\right) \in \mathbb{R}^{2}, m \in \mathbb{N}$. Lemma 2.3 implies that infinitely many of the constants $\lambda_{m}$ are different. Then we may assume that all the constants $\lambda_{m}$ are different. Now we have two cases to consider:

Case 1: There exists a subsequence of $\left(\lambda_{m}\right)$ that we still denote under $\left(\lambda_{m}\right)$ such that $\lambda_{m} \rightarrow \pm \infty$ as $m \rightarrow \infty$.

Since the problem is invariant under translation we can assume that $c_{1}(h)=$ $c_{-1}(h)=0$. We have

$$
c_{2 p+1}(\rho)=0 \quad \forall p \in \mathbb{Z}
$$


Indeed, suppose the contrary. Then there exists $p \geq 1$ such that $c_{2 p+1}(\rho) \neq 0$. Suppose first that $c_{3}(\rho) \neq 0$. By Lemma 2.2(1) we have

$$
\lambda_{m} c^{2}+\mu_{m}^{2}+\frac{b}{2 \pi \overline{c_{3}(\rho)}}=0 \quad \forall m \in \mathbb{N} .
$$

(4.2) implies that $\left|\mu_{m}\right| \rightarrow+\infty$ and $\lambda_{m} \rightarrow-\infty$ as $m \rightarrow \infty$. Then using (4.2) and Lemma 2.1 we get

$$
A=B_{1,2 p}=0 \quad \forall p \geq 2 .
$$

Since

$$
A=-2 \pi c \overline{c_{2}\left(h^{\prime}\right)}=4 i \pi c \overline{c_{2}(h)}
$$

and

$$
B_{1,2 p}=(-1)^{p-1} 2 \pi c \overline{c_{2 p}\left(h^{\prime}\right)}=(-1)^{p} 4 i p \pi c \overline{c_{2 p}(h)},
$$

we deduce that

$$
c_{2 n}(h)=0 \quad \forall n \in \mathbb{Z} \backslash\{0\} .
$$

But (4.6) implies that $\Omega$ is of constant width and we reach a contradiction. Now if $c_{3}(\rho)=0$, there exists $p \geq 2$ such that

$$
c_{2 j+1}(\rho)=0, \quad j=1, \ldots, p-1, \quad \text { and } \quad c_{2 p+1}(\rho) \neq 0 .
$$

By Lemma 2.2(2), $\mu_{m}$ satisfies a polynomial equation of degree 2 with coefficients depending on $\lambda_{m}$. It is quite easy to see that $\left|\mu_{m}\right| \rightarrow+\infty$ as $m \rightarrow \infty$. Then we deduce that

from which we derive

$$
\frac{\lambda_{m}}{\mu_{m}^{2}} c^{2}+1 \rightarrow 0 \quad \text { as } m \rightarrow \infty
$$

$$
\lambda_{m} c^{2}+\mu_{m}^{2}+\frac{(-1)^{p}}{2 \pi \overline{c_{2 p+1}(\rho)}}\left(\gamma_{1,2 p+1}-c^{2} \alpha_{2,2 p+1}\right) \rightarrow 0
$$

as $m \rightarrow \infty$ because $\beta_{1,2 q+1}=0$ for $q \geq 2$. Using (4.7) and Lemma 2.1 we deduce that (4.3) holds and we conclude as before. Thus (4.1) is proved.

Now we claim that there exists $p \in \mathbb{N} \backslash\{0\}$ such that

$$
\int_{0}^{L}\left(x_{1} \nu_{2}-x_{2} \nu_{1}\right)\left(\nu_{1}+i \nu_{2}\right)^{2 p} d s \neq 0
$$

Indeed, suppose the contrary. Then

$$
\int_{0}^{L}\left(x_{1} \nu_{2}-x_{2} \nu_{1}\right)\left(\nu_{1}+i \nu_{2}\right)^{2 p} d s=-2 \pi \overline{c_{2 p}\left(h^{\prime} \rho\right)}=0 \quad \forall p \in \mathbb{Z} .
$$


Using (4.1) and the fact that $c_{n}(\rho)=\left(1-n^{2}\right) c_{n}(h)$ for $n \in \mathbb{Z}$, we can write

$$
\begin{aligned}
c_{2 p+1}\left(h^{\prime} \rho\right) & =\sum_{j=-\infty}^{+\infty} c_{2 p+1-j}\left(h^{\prime}\right) c_{j}(\rho)=\sum_{j=-\infty}^{+\infty} c_{2(p-j)+1}\left(h^{\prime}\right) c_{2 j}(\rho) \\
& =i \sum_{j=-\infty}^{+\infty}(2(p-j)+1) c_{2(p-j)+1}(h) c_{2 j}(\rho)=0
\end{aligned}
$$

for all $p \in \mathbb{Z}$ (recall that $c_{1}(h)=c_{-1}(h)=0$ ). Therefore we have

$$
c_{n}\left(h^{\prime} \rho\right)=0 \quad \forall n \in \mathbb{Z},
$$

hence $h^{\prime} \rho=0$. Since $\rho$ is positive we deduce that $h$ is constant, contrary to (iii), and our claim is proved.

Now assume that $c_{2}\left(h^{\prime} \rho\right) \neq 0$. Then Lemma 2.1(1) implies that

$$
\lambda_{m} c^{2}+\mu_{m}^{2}+\frac{\overline{c_{2}\left(h^{\prime}\right)}}{\overline{c_{2}\left(h^{\prime} \rho\right)}} c \mu_{m}-\frac{B}{2 \pi \overline{c_{2}\left(h^{\prime} \rho\right)}}=0 \quad \forall m \in \mathbb{N} .
$$

Therefore $\left|\mu_{m}\right| \rightarrow+\infty$ as $m \rightarrow \infty$. Using (4.8), Lemma 2.1(2) and (4.5) we deduce that

$$
c_{2 p}\left(h^{\prime}\right)=\frac{c_{2}\left(h^{\prime}\right)}{c_{2}\left(h^{\prime} \rho\right)} c_{2 p}\left(h^{\prime} \rho\right) \quad \forall p \geq 2 .
$$

Clearly (4.9) also holds for $p=0,1$. Since $c_{2 p+1}\left(h^{\prime}\right)=c_{2 p+1}\left(h^{\prime} \rho\right)=0$ for all $p \in \mathbb{Z}$, we conclude that

$$
h^{\prime}=\frac{c_{2}\left(h^{\prime}\right)}{c_{2}\left(h^{\prime} \rho\right)} h^{\prime} \rho .
$$

It is quite elementary to see that (4.10) implies that $\rho$ is constant, contradicting (iii).

Now, as $c_{2}\left(h^{\prime} \rho\right)=0$, there exists $p \geq 2$ such that

$$
c_{2 j}\left(h^{\prime} \rho\right)=0, \quad j=1, \ldots, p-1, \quad \text { and } \quad c_{2 p}\left(h^{\prime} \rho\right) \neq 0 .
$$

By Lemma 2.1(2), $\mu_{m}$ satisfies a polynomial equation of degree 2 with coefficients depending on $\lambda_{m}$. Clearly $\left|\mu_{m}\right| \rightarrow+\infty$ as $m \rightarrow \infty$. Then we deduce that

$$
\frac{\lambda_{m}}{\mu_{m}^{2}} c^{2}+1 \rightarrow 0 \quad \text { as } m \rightarrow \infty
$$

from which we derive

$$
\lambda_{m} c^{2}+\mu_{m}^{2}+(-1)^{p-1} \frac{B_{1,2 p}}{2 \pi \overline{c_{2 p}\left(h^{\prime} \rho\right)}} \mu_{m}+\frac{(-1)^{p-1}}{2 \pi \overline{c_{2 p}\left(h^{\prime} \rho\right)}}\left(C_{1,2 p}-c^{2} A_{2,2 p}\right) \rightarrow 0
$$


as $m \rightarrow \infty$. Since $\left|\mu_{m}\right| \rightarrow+\infty$, (4.11) and Lemma 2.1 imply that

$$
A=0 \quad \text { and } \quad B_{1,4}=\cdots=B_{1,2 p-2}=0 \quad \text { if } p \geq 3 .
$$

Then using (4.12), Lemma 2.1(2) and (4.5) we deduce that

$$
c_{2 q}\left(h^{\prime}\right)=\frac{c_{2 p}\left(h^{\prime}\right)}{c_{2 p}\left(h^{\prime} \rho\right)} c_{2 q}\left(h^{\prime} \rho\right) \quad \forall q \geq p+1 .
$$

Since (4.13) also holds for $q=0, \ldots, p$, we conclude as before.

Case 2: The sequence $\left(\lambda_{m}\right)$ is bounded.

Let $\left(\varepsilon_{j}\right)$ and $\left(\varphi_{j}\right), j \in \mathbb{N}$, denote the eigenvalues and a complete orthonormal system of real eigenvectors for $-\Delta$ with Dirichlet boundary conditions. For any $z \in \mathbb{C} \backslash\left\{\varepsilon_{j} ; j \in \mathbb{N}\right\}$ we define $v_{z}$ as the solution of

$$
\Delta v_{z}+z v_{z}+1=0 \quad \text { in } \Omega, \quad v_{z}=0 \quad \text { on } \partial \Omega
$$

Since

$$
\int_{\partial \Omega} \frac{\partial v_{z}}{\partial n} d \sigma(x)=\sum_{j=0}^{+\infty} \frac{\varepsilon_{j}}{z-\varepsilon_{j}}\left(\int_{\Omega} \varphi_{j} d x\right)^{2}
$$

the function

$$
z \mapsto \int_{\partial \Omega} \frac{\partial v_{z}}{\partial n} d \sigma(x)
$$

is meromorphic on $\mathbb{C}$, nontrivial, and has a pole at $\varepsilon_{j}$ if and only if the corresponding eigenspace is not orthogonal to the constants. Now we define the function

$$
\mu(z)=\frac{c|\partial \Omega|}{\int_{\partial \Omega} \frac{\partial v_{z}}{\partial n} d \sigma(x)} .
$$

Lemma 4.1. In the above setting $\mu$ is a meromorphic function on $\mathbb{C}$ and $\mu\left(\lambda_{m}\right)=$ $\mu_{m}$ for every $m \in \mathbb{N}$ such that $\lambda_{m} \in \mathbb{R} \backslash\left\{\varepsilon_{j} ; j \in \mathbb{N}\right\}$

Proof. $\mu$ is clearly a meromorphic function on $\mathbb{C}$. If $u_{m}$ is a solution of (1.1)-(1.3) corresponding to $\left(\lambda_{m}, \mu_{m}\right)$, where $\lambda_{m} \in \mathbb{R} \backslash\left\{\varepsilon_{j} ; j \in \mathbb{N}\right\}$, then $u_{m}=\mu_{m} v_{\lambda_{m}}$. Therefore

$$
c=\mu_{m} \frac{\partial v_{\lambda_{m}}}{\partial n}
$$

and we get $\mu\left(\lambda_{m}\right)=\mu_{m}$.

Now, by taking a subsequence if necessary, we may assume that $\lambda_{m} \in \mathbb{R} \backslash$ $\left\{\varepsilon_{j} ; j \in \mathbb{N}\right\}$ for all $m \in \mathbb{N}$ and that there exists $\lambda_{\star} \in \mathbb{R}$ such that

$$
\lambda_{m} \rightarrow \lambda_{\star} \quad \text { as } m \rightarrow \infty .
$$


With the notations of Lemmas 2.1 and 2.2, for each $p \geq 1$ we define meromorphic functions on $\mathbb{C}$ by setting

$$
\begin{aligned}
F_{1}(z)= & \left(c^{2} z+\mu(z)^{2}\right) \int_{0}^{L}\left(x_{1} \nu_{2}-x_{2} \nu_{1}\right)\left(\nu_{1}+i \nu_{2}\right)^{2} d s+A \mu(z)+B, \\
G_{1}(z)= & \left(c^{2} z+\mu(z)^{2}\right) \int_{0}^{L}\left(\nu_{1}+i \nu_{2}\right)^{3} d s+a \mu(z)+b, \\
F_{p}(z)= & (-1)^{p} z^{p-1}\left(c^{2} z+\mu(z)^{2}\right) \int_{0}^{L}\left(x_{1} \nu_{2}-x_{2} \nu_{1}\right)\left(\nu_{1}+i \nu_{2}\right)^{2 p} d s \\
& +\mu(z)^{2} \sum_{j=2}^{p} A_{j, 2 p} z^{p-j}+\mu(z) \sum_{j=1}^{p} B_{j, 2 p} z^{p-j}+\sum_{j=1}^{p} C_{j, 2 p} z^{p-j}, \\
G_{p}(z)= & (-1)^{p} z^{p-1}\left(c^{2} z+\mu(z)^{2}\right) \int_{0}^{L}\left(\nu_{1}+i \nu_{2}\right)^{2 p+1} d s \\
& +\mu(z)^{2} \sum_{j=2}^{p} \alpha_{j, 2 p+1} z^{p-j}+\mu(z) \sum_{j=1}^{p} \beta_{j, 2 p+1} z^{p-j}+\sum_{j=1}^{p} \gamma_{j, 2 p+1} z^{p-j},
\end{aligned}
$$

if $p \geq 2$, where $\mu$ is defined in (4.14). By Lemmas 2.1, 2.2 and 4.1, for every $p \geq 1$, we have

$$
F_{p}\left(\lambda_{m}\right)=G_{p}\left(\lambda_{m}\right)=0 \quad \forall m \in \mathbb{N} .
$$

Notice that, for each $p \geq 1$, the poles of $F_{p}$ and $G_{p}$ are included in $\left\{\varepsilon_{j} ; j \in \mathbb{N}\right\}$. Since a nontrivial meromorphic function defined on all of $\mathbb{C}$ except at its poles cannot have a sequence of zeros with a finite limit point, we deduce that $F_{p} \equiv 0$ and $G_{p} \equiv 0$ for all $p \geq 1$. Then there exists a sequence $\left(\tilde{\lambda}_{m}\right)$ increasing to $+\infty$ in $\mathbb{R} \backslash\left\{\varepsilon_{j} ; j \in \mathbb{N}\right\}$ such that $F_{p}\left(\tilde{\lambda}_{m}\right)=G_{p}\left(\tilde{\lambda}_{m}\right)=0$ for $p \geq 1$ and $m \in \mathbb{N}$. Now we can argue as in Case 1.

\section{$\S 5$. Concluding remarks}

The following theorem was proved in [13, Theorem 3.2 p. 1198].

Theorem 5.1. Let $\Omega \subset \mathbb{R}^{2}$ be a bounded convex open set of class $C^{3, \alpha}(\alpha \in(0,1])$. Assume that $\Omega$ is not of constant width and that $\Omega$ has the Schiffer property. Let $\psi \in C^{1}(\partial \Omega)$ be such that:

(i) $\psi$ is not identically constant;

(ii) $\psi$ has at most countably many zeros.

Then there exist at most finitely many different pairs of coefficients $\left(\lambda_{m}, \mu_{m}\right) \in \mathbb{R}^{2}$ such that the Cauchy problem

$$
\Delta u+\lambda_{m} u+\mu_{m}=0 \quad \text { in } \Omega, \quad u=0 \quad \text { and } \quad \frac{\partial u}{\partial n}=\psi \quad \text { on } \partial \Omega,
$$

has a solution. 
Using a completely different approach we proved the same theorem in [5], Theorem 1.3 p. 774] under slightly different assumptions. However the conditions there imply that $\Omega$ is not of constant width and that $\Omega$ has the Schiffer property. On the other hand we only assume in [5] that $\psi \in C^{\infty}(\partial \Omega)$ is not identically constant. Theorem 1.1 shows that this assumption is not necessary.

The proof of Theorem 1.1 in Case 1 shows that $\lambda_{m} \rightarrow+\infty$ cannot occur. Then the arguments in Case 2 easily lead to a contradiction.

We have seen in the proof of Theorem 1.1 that if (1.1)-(1.3) has a solution corresponding to a sequence of infinitely many different pairs $\left(\lambda_{m}, \mu_{m}\right) \in \mathbb{R}^{2}$, $m \in \mathbb{N}$, and if there exists $p \geq 1$ such that $c_{2 p+1}(\rho) \neq 0$, then $\Omega$ is of constant width. A class of smooth bounded convex sets of constant width for which there exist at most finitely many different pairs of coefficients $\left(\lambda_{m}, \mu_{m}\right) \in \mathbb{R}^{2}$ such that (1.1)-(1.3) has a solution is given in [7, Proposition 6.2 p. 118]. We have the following stronger result, where we use the notations introduced in Section 2.

Theorem 5.2. Let $\Omega \subset \mathbb{R}^{2}$ be a bounded convex open set satisfying the following conditions:

(i) $\partial \Omega$ is a $C^{\infty}$ curve with positive curvature;

(ii) $\Omega$ has the Schiffer property;

(iii) $\Omega$ has constant width;

(iv) $\left\{p \in \mathbb{N} \backslash\{0\} ; c_{2 p+1}(\rho) \neq 0\right\}$ is finite.

Let $c$ be a nonzero constant. Then there exist at most finitely many different pairs of coefficients $\left(\lambda_{m}, \mu_{m}\right) \in \mathbb{R}^{2}$ such that the Cauchy problem

$$
\Delta u+\lambda_{m} u+\mu_{m}=0 \quad \text { in } \Omega, \quad u=0 \quad \text { and } \quad \frac{\partial u}{\partial n}=c \quad \text { on } \partial \Omega,
$$

has a solution.

Proof. We shall need a simple lemma proved in [7, Lemma 6.1 p. 118].

Lemma 5.1. In the setting of Theorem 5.2, for all $p \in \mathbb{N}$, there exists $q \geq p$ such that $c_{2 q+1}(1 / \rho) \neq 0$.

Now as in the proof of Theorem 1.1 we are led to Case 1. Assume first that $c_{3}(\rho) \neq 0$. Then (4.2) holds, hence $\left|\mu_{m}\right| \rightarrow+\infty$ and $\lambda_{m} \rightarrow-\infty$ as $m \rightarrow \infty$. Using Lemma 2.2(2) we deduce that

$$
c_{2 n+1}\left(\frac{1}{\rho}\right)=\frac{2}{n(n+1)} \frac{c_{3}(1 / \rho)}{c_{3}(\rho)} c_{2 n+1}(\rho) \quad \forall n \geq 2 .
$$

(Notice that (5.1) also holds for $n=1$.) Then Lemma 5.1 and (iv) give a contradiction. Now if $c_{3}(\rho)=0$, as in the proof of Theorem 1.1 we arrive at (4.7). Since 
for all $n \geq 2$,

$$
\gamma_{1,2 n+1}-c^{2} \alpha_{2,2 n+1}=(-1)^{n} 4 \pi n(n+1) c^{2} \overline{c_{2 n+1}(1 / \rho)},
$$

using Lemma 2.2(2) we deduce that if $c_{2 j+1}(\rho)=0, j=1, \ldots, p-1$, and $c_{2 p+1}(\rho)$ $\neq 0$, then

$$
c_{2 n+1}\left(\frac{1}{\rho}\right)=\frac{p(p+1)}{n(n+1)} \frac{c_{2 p+1}(1 / \rho)}{c_{2 p+1}(\rho)} c_{2 n+1}(\rho) \quad \forall n \geq p+1
$$

(clearly (5.2) also holds for $n=p$ and in fact also for $n=1, \ldots, p-1$ ), and we conclude as before. The arguments in Case 2 are the same as in the proof of Theorem 1.1.

Notice that the class $\mathcal{B}$ in [7, Definition 1.1 p. 94 and Proposition 6.2 p. 118] is strictly contained in the class defined in Theorem 5.2. Indeed, if $c_{3}(\rho) \neq 0$, then $\Omega$ has the Schiffer property: see Remark 4 or [7, Proposition 6.1 p. 117]. However there are plenty of smooth domains of constant width having the Schiffer property and such that $c_{3}(\rho)=0$. Let us give an example. Let $r>72$ and define

$$
h(\theta)=r+\cos 5 \theta+\cos 7 \theta, \quad \theta \in[0,2 \pi] .
$$

Then $h$ is of class $C^{\infty}$ and has period $2 \pi$. Since

$$
\rho(\theta)=r-24 \cos 5 \theta-48 \cos 7 \theta>0, \quad 0 \leq \theta \leq 2 \pi,
$$

$h$ must be the support function of a convex set $\Omega$. Clearly $c_{3}(\rho)=0$. Since $h(\theta)+$ $h(\theta+\pi)=2 r, \Omega$ has constant width. Assume that (1.1)-(1.3) has a solution when $c=0$ and $\mu=1$. In Lemma 2.1(1) we have $A=B=0$ and

$$
\int_{0}^{L}\left(x_{1} \nu_{2}-x_{2} \nu_{1}\right)\left(\nu_{1}+i \nu_{2}\right)^{2} d s=-\int_{0}^{2 \pi} h^{\prime}(\theta) \rho(\theta) e^{2 i \theta} d \theta=36 i \pi \neq 0,
$$

and we reach a contradiction.

Finally let $u$ be a solution of (1.1)-(1.3). With the notations of Section 4 , the eigenvalue $\varepsilon_{0}$ is simple and the eigenfunction $\varphi_{0}$ is of constant sign. Integrating by parts we obtain

$$
\varepsilon_{0} \int_{\Omega} u \varphi_{0} d x=-\int_{\Omega} u \Delta \varphi_{0} d x=-\int_{\Omega} \varphi_{0} \Delta u d x=\lambda \int_{\Omega} u \varphi_{0} d x+\mu \int_{\Omega} \varphi_{0} d x .
$$

If $\lambda=\varepsilon_{0}$, we deduce that $\mu=0$. Then by the classical result of Serrin [12, $\Omega$ is a disk.

\section{References}

[1] C. A. Berenstein, An inverse spectral problem and its relation to the Pompeiu problem, J. Anal. Math. 37 (1980), 128-144. Zbl 0449.35024 MR 0583635 
[2] L. Brown and J.-P. Kahane, A note on the Pompeiu problem for convex domains, Math. Ann. 259 (1982), 107-110. Zbl 0464.30035 MR 0656655

[3] R. Dalmasso, A note on the Schiffer conjecture, Hokkaido Math. J. 28 (1999), 373-383. Zbl 0931.35042 MR 1698430

[4] A new result on the Pompeiu problem, Trans. Amer. Math. Soc. 352 (2000), 27232736. Zbl 0940.35145 MR 1694284

[5] _ An inverse problem for an elliptic equation with an affine term, Math. Ann. 316 (2000), 771-792. Zbl 0958.35021 MR 1758453

[6] _ A uniqueness result for an inverse problem, Ann. Polon. Math. 84 (2004), 61-66. Zbl pre02120246 $\mid$ MR 2112015

[7] , An inverse problem for an elliptic equation, Publ. RIMS Kyoto Univ. 40 (2004), 91-123. Zbl 1134.35401 MR 2030071

[8] H. Flanders, A proof of Minkowski's inequality for convex curves, Amer. Math. Monthly $\mathbf{7 5}$ (1968), 581-593. Zbl 0162.25803 MR 0233287

[9] R. Garofalo and F. Segala, Univalent functions and the Pompeiu problem, Trans. Amer. Math. Soc. 346 (1994), 137-146. Zbl 0823.30027 MR 1250819

[10] G. M. L. Gladwell and N. B. Willms, Saddle points and overdetermined problems for the Helmholtz equation, Z. Angew. Math. Phys. 45 (1994), 1-26. Zbl 0793.35065 MR 1259523

[11] M. A. Hurwitz, Quelques applications géométriques des séries de Fourier, Ann. Scuola Norm. Sup. Pisa 19 (1902), 357-408. JFM 33.0599.02 MR 1509016

[12] J. Serrin, A symmetry problem in potential theory, Arch. Ration. Mech. Anal. 43 (1971), 304-318. Zbl 0222.31007 MR 0333220

[13] M. Vogelius, An inverse problem for the equation $\Delta u=-c u-d$, Ann. Inst. Fourier (Grenoble) 44 (1994), 1181-1209. Zbl 0813.35136 MR 1306552

[14] G. N. Watson, A treatise on the theory of Bessel functions, 2nd ed., Cambridge Univ. Press, 1962. Zbl 0849.33001 MR 1349110

[15] S. A. Williams, A partial solution to the Pompeiu problem, Math. Ann. 223 (1976), 183-190. Zbl 0329.35045 MR 0414904

[16] Analyticity of the boundary for Lipschitz domains without the Pompeiu property, Indiana Univ. Math. J. 30 (1981), 357-369. Zbl 0439.35046 MR 0611225

[17] _ Boundary regularity for a family of overdetermined problems for the Helmholtz equation, J. Math. Anal. Appl. 274 (2002), 296-304. Zbl 1049.35081 MR 1936699

[18] S.-T. Yau, Problem section, in Seminar on differential geometry, S.-T. Yau (ed.), Ann. of Math. Stud. 102, Princeton Univ. Press, Princeton, 1982, 669-706. Zbl 0479.53001 MR 0645762

[19] L. Zalcman, Supplementary bibliography to "A bibliographic survey of the Pompeiu problem", in Radon transforms and tomography (South Hadley, MA, 2000), Contemp. Math. 278, Amer. Math. Soc., 2001, 69-74. Zbl 1129.26300||MR 1851479 